\title{
Needle Stick Injuries in a Tertiary Care Hospital in Bihar - Current Indian Scenario
}

\author{
Manasij Mitra1, Maitraye Basu², Gautam Sarker³ \\ ${ }^{1}$ Department of Anaesthesiology, MGM Medical College and LSK Hospital, Kishanganj, \\ Bihar, India. ${ }^{2}$ Department of Biochemistry, MGM Medical College and LSK Hospital, \\ Kishanganj, Bihar, India. ${ }^{3}$ Department of Community Medicine, MGM Medical College \\ and LSK Hospital Kishanganj, Bihar, India.
}

\section{ABSTRACT}

\section{BACKGROUND}

Needle Stick Injuries caused by hypodermic needles, intravenous stylets or other sharps are an occupational hazard as these can lead to transmission of bloodborne pathogens. This study was conducted to understand the pattern of Needle Stick Injuries (NSIs) in our hospital settings.

\section{METHODS}

An observational retrospective study was conducted in a 600 bedded Tertiary Care Hospital in Kishanganj, Bihar. A total of 87 NSIs were encountered during the study period from April 2018 to March 2019 using NACO guidelines. Data was entered into a computer-based spreadsheet for analysis using SPSS statistical software, version 19. The statistical tests applied included descriptive statistics and Chisquare tests to observe the association, if any.

\section{RESULTS}

Direct HCWs like Nursing Professionals and Doctors sustained more NSIs. Staff with $>2$ years of experience sustained less NSIs. Staff who received training on awareness and prevention of NSIs within the last 6 months sustained less NSIs. Introduction of engineered safety devices brought about an overall reduction in the NSI rate per 100 occupied beds from $7.83 \%$ to $6.67 \%$, a reduction by $14.81 \%$. This reduction in NSI rate was statistically significant ( $p$ value $=0.003$ ).

\section{CONCLUSIONS}

There is a definite role of experienced staff and frequent trainings in reducing NSIs. This study brought out another very significant observation not reviewed previously in Indian settings, the use of engineered safety devices in bringing down NSIs. Certain policy recommendations at the National Level to strengthen monitoring and surveillance to safeguard HCWs from occupational exposure to bloodborne pathogens is also felt.

\section{KEY WORDS}

Needle Stick Injury, Health Care Workers, Occupational Hazard, Engineered Safety Devices

\author{
Corresponding Author: \\ Dr. Maitraye Basu, \\ Department of Biochemistry, \\ MGM Medical College \& LSK Hospital, \\ Kishanganj-855107, Bihar, India. \\ E-mail: maitraye.basu@gmail.com
}

DOI: $10.14260 /$ jemds/2020/65

Financial or Other Competing Interests: None.

How to Cite This Article:

Mitra M, Basu M, Sarker G. Needle stick injuries in a tertiary care hospital in Biharcurrent Indian scenario. J. Evolution Med. Dent. Sci. 2020;9 (05):289-294, DOI: 10.14260/jemds/2020/65

Submission 02-12-2019,

Peer Review 11-01-2020,

Acceptance 17-01-2020,

Published 03-02-2020. 


\section{BACKGROUND}

A needle stick injury (NSI), commonly encountered by healthcare professionals, is an accidental percutaneous piercing wound typically set by a needle point, like hypodermic needles, intravenous stylets or other sharp objects like scalpels and suture needles. These injuries can occur anytime while handling needles or other sharps while providing care to patients.[1] Punctures by contaminated needles can inject hazardous fluids into the body. Though there is a chance for injection of hazardous drugs, but contact with infectious fluids, especially blood, is the greatest concern. Even small amount of infectious fluid can spread certain diseases effectively.[2] Such injuries are thus an occupational hazard in the healthcare community as these injuries may lead to transmission of bloodborne pathogens such as hepatitis B virus (HBV), hepatitis C virus (HCV), or human immunodeficiency virus (HIV). In rare isolated events needle stick injuries have transmitted many other diseases involving viruses, bacteria, fungi, and other microorganisms. These bear testimony that needle stick injuries can have serious consequences.[3]

The incidence of occupational exposures to blood borne pathogens through needles and other sharps was highlighted as 19.46 per one hundred occupied beds per annum as per the Exposure Prevention Information Network 2011 report. [4] The risk of infection after exposure to infected blood varies by bloodborne pathogen. WHO reports in the World Health Report 2002, that of the 35 million health-care workers, 2 million experience percutaneous exposure to infectious diseases each year. It further notes that $37.6 \%$ of Hepatitis $B$, $39 \%$ of Hepatitis C and $4.4 \%$ of HIV/AIDS in Health-Care Workers around the world are due to needle stick injuries.[5] It is most likely because the hepatitis B virus may survive on environmental surfaces for more than a week, indirect exposure can occur via contaminated inanimate objects.[6]

As per a report from the Centers for Disease Control and Prevention (CDC) in the United States from a study with data collected from 1995 to 2007, needle stick injuries can occur at every stage of the use of their use, disassembly, or disposal. The report further concluded that equipment design, nature of the procedure, condition of work, staff experience, recapping, and disposal have all been mentioned as factors that influence these occurrences.[7] Occupational exposures are not uncommon in the developing world. However, underreporting poses serious concerns in the developing world. This has got serious implications and bearings which can be deterrents for injured HCWs from receiving post exposure prophylaxis (PEP) against HIV, which is shown to be $80 \%$ effective in preventing HIV infection in these subjects. ${ }^{[8,9]}$

Data on the prevalence and attributes of NSIs in different healthcare settings in India is scare. [10] Preventing needle stick injuries is the best approach to prevent occupational exposure and transmission of bloodborne pathogens in healthcare workers and is an important part of any bloodborne pathogen prevention program in the workplace.[11] With the above background the study was conducted to understand the pattern of NSIs in our hospital settings. The objectives of the study were to understand the correlates of Needle Stick Injury in our hospital settings and whether introduction of engineered safety devices had an impact on the Needle Stick Injury rate.

\section{METHODS}

An observational retrospective study which was conducted in MGM Medical College and LSK Hospital, Kishanganj, Bihar, a 600 bedded Tertiary care Hospital. A total of 87 NSIs were the participants during the study period from April 2018 to March 2019. A clearance was obtained from Institutional Ethics Committee before initiation of the study. There is a protocol of reporting and handling a Needle Stick Injury round the clock through a Needle Stick Injury Report Form. The hospital has an integrated post exposure blood testing facility and (post exposure prophylaxis) PEP Program executed through the Emergency Medical Officer (EMO) based on the National AIDS Control Organization of India (NACO) guidelines.[12] The standard proforma for tests as prescribed in the NACO guidelines for each occupational exposure is imbibed in the existing protocol and followed. The hospital infection control team tracks each exposed HCW (health care worker) at the end of 6 weeks, 3 months and 6 months.

There is also an existing approach to prevention of NSIs through awareness generation, training, ensuring continuous supply, availability and use of recommended Personal Protective Equipment (PPEs) like latex free gloves and heavy duty gloves while handling blood and other body fluids or biomedical wastes, banning of needle recapping, using dedicated sharps containers and colour coded bins as per the BMW Management Rules 2016.[13] There are regular classroom and on-job training sessions for existing employees at least once a year and also once a month for newly joined staff through the Induction program. As a protocol and routine practice, whenever, a staff sustains a needle stick injury, the NSI Report Form is filled up by the Staff or his/her supervisor and gets routed to the Infection Control Officer within 48 hours following Risk Stratification as per NACO guidelines ${ }^{[14]}$ by the EMO. Baseline investigations are done in compliance with NACO guidelines following counselling by authorized personnel and written informed consent. Baseline investigations for the HCWs include Serology for HIV 1 and 2, Hepatitis B and Hepatitis C, Liver Function Tests and Anti HBs Titre. The serum samples of patients from whom the HCWs got exposed, if known, are also tested for HIV, HBV, and HCV with viral load, if screening tests are positive.

The NSI Report Form is a Semi structured Questionnaire which includes information on Employee Id, Staff Category, Sex, Education Level, years of experience, place of occurrence of the NSIs, use of PPEs at the time of NSI, type of the needle causing the injuries, mechanism of Injury, status of the source of injury (unknown/known), whether hospital protocol was followed after NSI, status of training and status of Hepatitis B Vaccination. The blood samples of the staff who sustained NSI as well as the source if known, is analysed in accredited Laboratory of the hospital using standard guidelines following "Zero billing" where the cost of the investigations are borne by the hospital.

Staff with anti-HBs titer $\geq 10 \mathrm{mIU} / \mathrm{mL}$ are considered as responders to vaccination and $<10 \mathrm{mIU} / \mathrm{mL}$ as non- 
responders and are subjected to further management as per established guidelines and status of the source. HCWs who get exposed to HIV seropositive patients or Hepatitis C infected patients are immediately referred to the in-house EMO/Infectious disease specialist. To track the seroconversion, all HCWs are counselled and advised to get tested again after 3 weeks, 3 months and finally after 6 months. There is a structured repository with details of each NSI incident, investigation reports and follow up reports as mentioned above with direct oversight by the Infection Control Team.

\section{Statistical Analysis}

Data for this study thus collected for the period from April 2018 to March 2019 was entered into a computer-based spreadsheet for analysis using SPSS statistical software (version 19) (IBM Corp., NY, USA). The statistical tests applied included descriptive statistics and Chi-square tests for testing the association, if any.

\section{RESULTS}

A total of 87 cases of accidental exposures to NSIs were reported during the period of Study. The mean age of the study group was 24.6 years (standard deviation \pm 4.2 ), range 19-38 years. Majority of the HCWs who sustained NSIs were females $71.26 \%$ (figure 1). Direct HCWs like Nursing Professionals and Doctors sustained more NSIs (59, 67.82\%) in comparison to Allied HCWs like Housekeeping staff, Technicians and full meaning HCAs $(28,32.18 \%)$. This difference is statistically significant ( $p$ value=0.006) However, when individual staff categories were analysed, nursing professionals namely staff nurse (47.13\%) and student nurse $(17.24 \%)$ constituted majority of the HCWs who sustained NSIs. Housekeeping staff $(24.14 \%)$ were the next highest in the category. The technicians constituted $5.75 \%$ of the staff who sustained NSIs, Doctors constituted $3.45 \%$ and HCAs another $2.3 \%$ of the staff who sustained NSIs (figure 2). Analysis of education level of the staff who sustained NSI revealed $27.59 \%$ staff studied up to Class 8 , $20.69 \%$ staff studied up to Class 12 and $51.72 \%$ staff were graduates. The graduates included the BSc nursing staff and the doctors (figure 3).

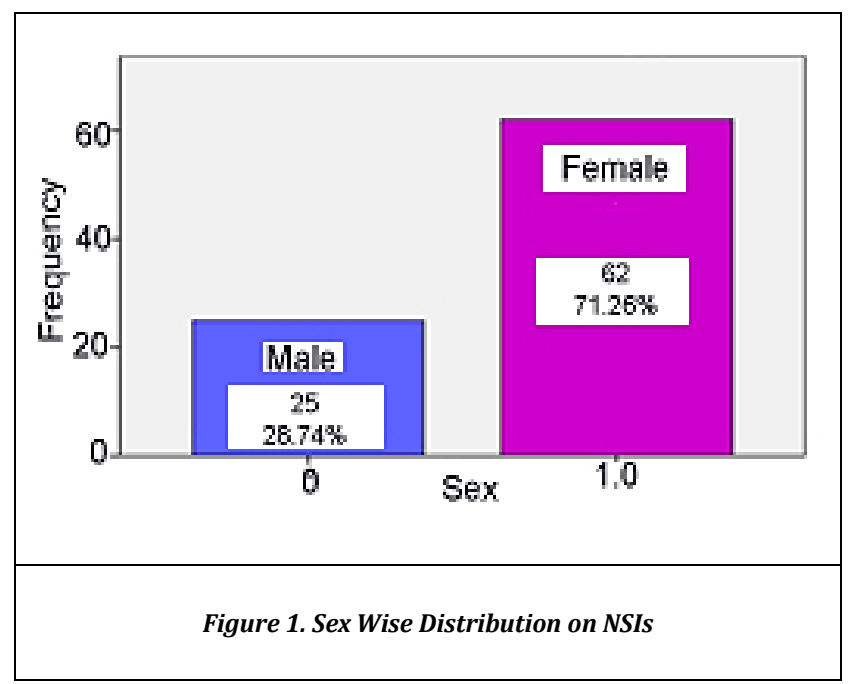

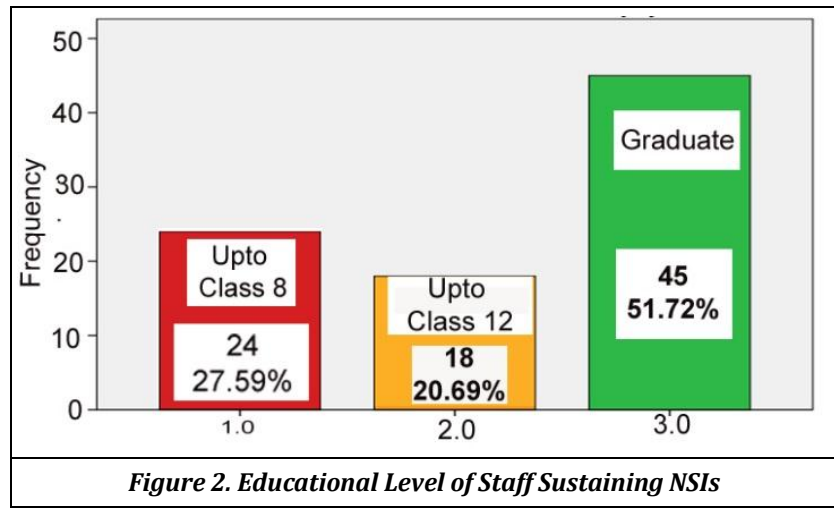
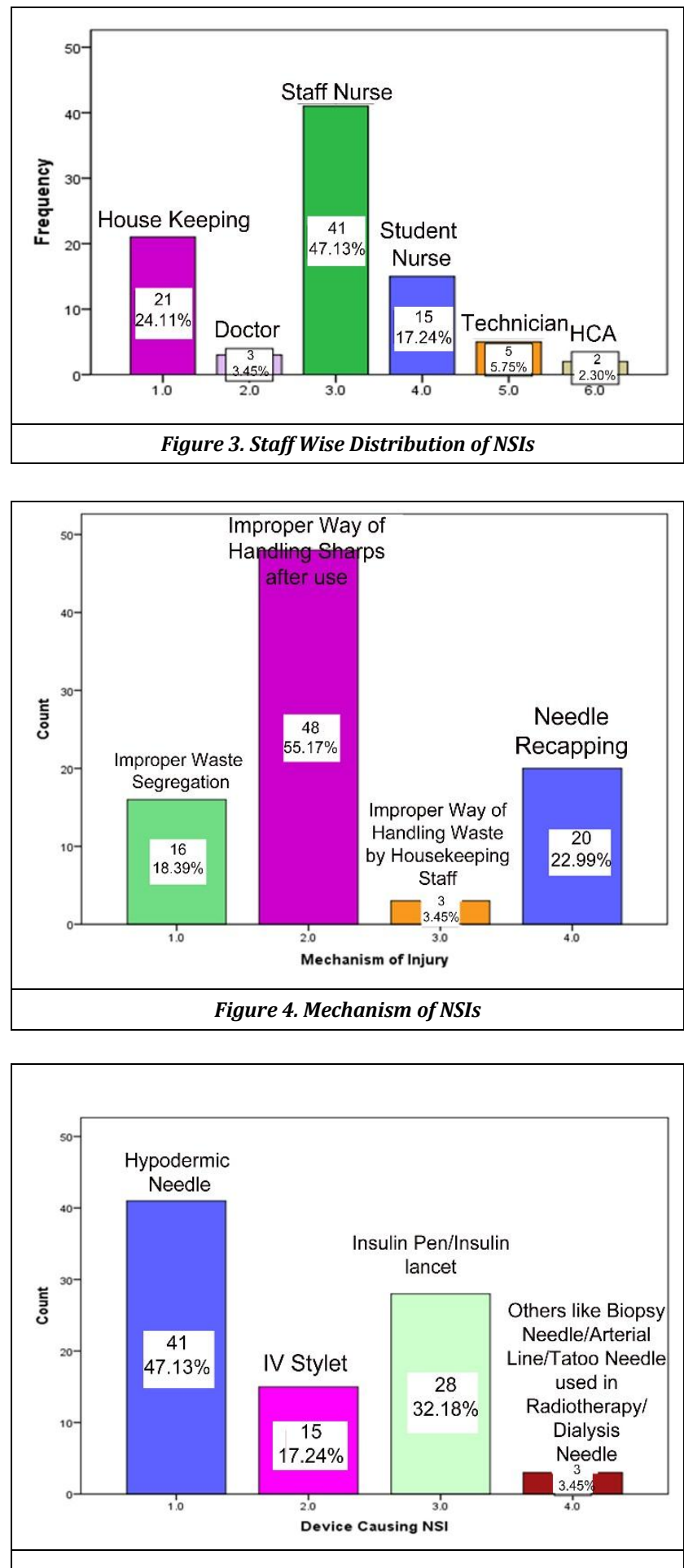

Figure 5. Types of Devices Causing NSIs 
Improper way of handling sharps after use contributed to $55.17 \%$, needle recapping contributed to $22.99 \%$, improper waste segregation contributed to $18.39 \%$ and improper way of handling waste by Housekeeping staff contributed to $3.45 \%$ NSIs (figure 4). Hypodermic syringe contributed to $47.13 \%$ of the NSIs, Insulin Pen/Insulin Lancet contributed to $32.18 \%$, IV stylets to another $17.24 \%$ while biopsy needles/arterial Lines/tattoo needle used in Radiotherapy/Dialysis needle contributed to another $3.45 \%$ of the devices (figure 5).

Analysis of the status of the source revealed that in 59.8\% cases source was traceable with negative serology. The remaining $40.2 \%$ source was either unknown $(25.3 \%)$ or Hepatitis B positive $(10.3 \%)$ or Hepatitis C positive $(3.4 \%)$, with only $1.2 \%$ HIV positive. Staff with $>2$ years of experience sustained less NSIs $(26,29.88 \%)$ in comparison to staff with less years of experience $(61,70.12 \%)$ and this difference is statistically significant ( $\mathrm{p}$ value 0.017 ). This is due to their skill in handling sharps which comes with years of experience. $(85,97.7 \%)$ of the Staff who sustained NSIs did not have any previous history of NSI in their career while $(2$, $2.3 \%$ ) of the staff reported previous history of NSIs. However, this difference was not statistically significant $(\mathrm{P}$ value= 0.28). Occurrence of NSIs was more in Emergency and Critical Care Units $(48,55.17 \%)$ in comparison to the Wards and Day Care units $(35,40.22 \%)$ and Procedure Areas and Operation Theatres $(4,4.61 \%)$ though this difference was not statistically significant ( $\mathrm{p}$ value $=0.42$ ).

Use of PPEs increased with Education Level of Staff. PPE use like Gloves was $(18,20.68 \%)$ in Staff with Education Level up to Class 8, $(24,27.58 \%)$ in Staff with Education Level up to Class 12 and $(45,51.74 \%)$ in Graduate Staff. This difference was statistically significant ( $p$ value $=0.015$ ). Staff who received training on awareness and prevention of NSIs within the last 6 months sustained less NSIs $(22,25.28 \%)$ against staff who never received any training or received training before 6 months $(65,74.72 \%)$ and this difference was statistically significant ( $\mathrm{p}=0.047)$. A need to increase the frequency of training on awareness and prevention of NSI from the current practice of once a year to twice a year is felt as staff who received training on awareness and prevention of NSI within 6 months sustained less NSIs.

All 87 staff who suffered NSI washed their hands with soap and water and followed the NSI protocol. Compliance to NSI protocol was cent percent in our study which can be due to the existing structured program and the stringent existing systems of monitoring and supervision in the study settings. Staff with complete vaccination status had Anti HepB titre $>10 \mathrm{mIU} / \mathrm{ml}(82,94.25 \%)$ while staff with incomplete vaccination status or unknown vaccination status had Anti HepB titre $<10 \mathrm{mIU} / \mathrm{ml}(5,5.75 \%)$. This difference was statistically significant $(\mathrm{P}$ value $=0.001)$. The mean Anti HepB Titre was $305.93 \mathrm{mIU} / \mathrm{ml}$ (standard deviation \pm 96.46). Engineered safety devices which are available in India were introduced from October 2018 with hands on training of the staff to make them at ease with use. There was round the clock vigilance from the Infection Control Team to ensure that the engineered safety devices were readily available for use and staff were confident in using them. This intervention brought about an overall reduction in the NSI rate per 100 occupied beds from $7.8 \%$ to $6.67 \%$, a reduction by $14.49 \%$
This reduction in NSI rate was statistically significant ( $p$ value $=0.003$ ).

At the end of 6 months, $82.76 \%$ staff completed the follow up. The seroconversion rate for Hepatitis B, Hepatitis C and HIV for the staff who completed the follow up was nil. However, $17.24 \%$ of the staff were lost to follow up due to their resignation and disassociation with the organisations.

\section{DISCUSSION}

Occupational exposures are not uncommon. Poor compliances to universal precautions is a risk factor for sharp injuries.[15] Prevalence of NSI and the risk factors associated vary among different HCW groups such as doctors and nurses depending on study settings.[16,17] Probability of having NSI is inversely proportional to the years of experience. This may be due to lack of experience, handling the heavy workload of patients under pressure situations and knowledge regarding injection safety guidelines.[18] Common areas are the Emergency, OT and the ICUs where there may be a tendency to hurry, work pressure and increased use of needles and sharps.[19] Studies in Indian scenario revealed, around onefourth of the vaccinated individuals had anti-HBs titers $<10$ $\mathrm{IU} / \mathrm{ml}$, which is the protective level. It can be due to incomplete vaccination or not taking the booster dose. There is enough National and International Literature to substantiate the fact.[20,21,22]

An additional challenge encountered with Needle Stick Injuries in Indian settings is the underreporting due lack of established protocol following an NSI, lack of proper training and awareness programs, excessive time required to follow protocol, negative stigmatization and perception of low risk for bloodborne pathogen infections. $40-75 \%$ of these injuries are not reported. Such unreported injuries are a serious problem and pose impediments for HCWs from receiving timely medical intervention and thus reduce the chances of transmission of the blood borne pathogens. These findings necessitate the need to formulate strategies to reduce such injuries in a systematic way and to improve reporting system so that timely and appropriate medical care can be delivered.[23,24] Over $80 \%$ of needlestick injuries are preventable through use of safer needle devices (CDC, 1997). This in conjunction with worker education and work practice controls, can reduce injuries by over $90 \%$ (Jagger, 1996).

Prevention is cost-effective. The cost of follow-up for an exposure as estimated is almost $\$ 3,000$ per needlestick injury, even when no infection occurs (Jagger, Bentley, \& Juillet, 1998). One case of serious infection by bloodborne pathogens can add up to $\$ 1$ million or more in expenditures for testing, follow-up, lost time, and disability payments (Pugliese \& Salahuddin, 1999).[25] The Occupational Safety and Health Administration (OSHA) published the original Bloodborne Pathogens Standard (BBP) in 1991.[26] This standard requires employers to take action to reduce employees' risk of exposure to bloodborne pathogens.

A comprehensive NSI prevention program would take into consideration policies and processes build upon recommended guidelines; implementing improved 
equipment design; establishing effective biomedical waste disposal systems; comprehensive employee training encompassing staff awareness on the risk of NSI; promoting use of PPE; mandatory hepatitis B vaccination for HCWs; nonrecapping or safe recapping procedures; surveillance programs that provide in-depth analysis of the injuries and ensuring injured workers receive proper treatment.[27,28]

In developed countries preventing needle stick injuries is considered a part of the "routine practices" used by healthcare workers.[29,30] The use of safety-engineered devices built with consideration to both the safety of the health care worker and the patient such as protected needle devices, or needle-free systems with self-sealing ports and syringes is encouraged. In certain developed nations the use of such safety devices is required by some jurisdictions.[31.32]

Studies and data on Needle Stick Injuries in Indian Healthcare Settings is scare. There are no national reporting systems for NSIs in India, but a report in 2006 showed that around $63 \%$ of the 3-6 billion injections given every year are unsafe. [33] This topic is gaining importance slowly in India. Also, the policy implications pertaining to the NSIs in the current Indian scenario has not been discussed through any study observations. ${ }^{[34,35]}$ As more and more hospitals in India are opting for national and international accreditations with an aim to improve the quality of cares, structured policies and programs on prevention and post exposure protocols exist in many hospitals today. All accrediting bodies give emphasis on the implementation of NSI protocols and occupational safety of the HCW. But in the absence of mandates and regulations in the country, many small hospitals and healthcare units operating as local clinics and nursing homes do not have any protocols in place. Thus, thousands of HCWs in the current Indian scenario are still helpless victims of NSIs and its aftereffects. The use of engineered safety devices are mandates in many countries. [36] Unfortunately, no such mandate exists in Indian settings and the use of engineered safety devices is left on the choice of the employer. In most circumstances, employers consider the immediate cost of the engineered safety devices which acts as a deterrent in its implementation.

\section{CONCLUSIONS}

There is a definite role of experienced staff and frequent trainings in reducing NSIs. Further this study brought out a very significant observation not studied previously in Indian settings, the use of engineered safety devices in bringing down NSIs. The study further highlights the need for framing structured policies at the National Level with mandates for enforcement of centralised reporting systems for NSIs and use of engineered safety devices as it exists in many developed countries like USA, Canada, Europe and also in many South East Asian countries to safeguard HCWs from occupational exposures to blood borne pathogens. There is also a further need for similar studies to understand the dynamics of the NSIs and role of engineered safety devices in reducing the NSI rates and the cost implications.
Limitations

The sample size of the study was very small as the nature of the study (observational retrospective study) did not allow to have a large sample size.

\section{REFERENCES}

[1] National Institute for Occupational Safety and Health. U.S. department of health and human services Public Health Service Centers for Disease Control and Prevention National Institute for Occupational Safety and Health. Alert: Preventing Needle Stick Injuries in Healthcare Settings. DHHS (NIOSH) Publication No. 2000-108 November 1999 https://www.cdc.gov/niosh/docs/2000-08/pdfs/2000108.pdf [Last cited on 01.11.2019]

[2] Needle stick and sharp injuries. Canadian Centre for Occupational Health and Safety. https://www.ccohs.ca/oshanswers/diseases/needlestic k_injuries.html [Last cited on 06.12.2019]

[3] WHO Guidelines on Drawing Blood: Best Practices in Phlebotomy. Geneva: World Health Organization: 2010. Annex D, Managing occupational exposure to hepatitis B, hepatitis $\mathrm{C}$ and HIV. https://www.ncbi.nlm.nih.gov/books/NBK138653/ [Last cited on 01.11.2019]

[4] Beltrami EM, Williams IT, Shapiro CN, et al. Risk and management of blood-borne infections in health care workers. Clin Microbiol Rev 2000;13 (3):385-407.

[5] The National Surveillance System for Healthcare Workers

$(\mathrm{NaSH})$. https://www.cdc.gov/nhsn/PDFs/NaSH/NaSH-Report6-2011.pdf [Last cited on 06.12.2019].

[6] Centers for Disease Control and Prevention: NIOSH Publications and Products. Preventing Needlestick Injuries in Health Care Settings (2000-108) [Last cited on 2019 Dec 6]. http://www.cdc.gov/niosh/docs/2000$108 /$

[7] Wilburn SQ. Needlestick and sharps injury prevention. Online J Issues Nurs 2004; 9 (3):5.

[8] Sriram S. Study of needle stick injuries among healthcare providers: evidence from a teaching hospital in India. J Family Med Prim Care 2019;8 (2):599-603.

[9] International Healthcare Worker Safety Center, University of Virginia. U.S. EPINet Sharps Injury and Blood and Body Fluid Exposure Surveillance Research Group. Sharps Injury Data Report: 2011. http://www.healthsystem.virginia.edu/pub/epinet/epin etdatareports.html\#CitingEPINet [Last cited on 01.11.2019].

[10] Occupational health. World Health Report 2002. http://www.who.int/occupational_health/topics/neelinj uries/en/index.html [Last cited on 01.11.2019]

[11] Canadian Centre for Occupational Health and Safety. Needlestick and Sharps Injuries. http://www.ccohs.ca/oshanswers/diseases/needlestick _injuries.html [Last cited on 01.11.2019]

[12] Post Exposure Prophylaxis (PEP)-NACO Guidelines. http://www.upsacs.in/pdf/GUIDELINES/PEP.pdf [Last cited on 06.12.2019] 
[13] Singhal L, Tuli AK, Gautam V. Biomedical waste management guidelines 2016: What's done and what needs to be done. Indian J Med Microbiol [serial online] 2017;35 (2):194-8.

[14] National Technical Guidelines on Antiretroviral Treatment. National Aids control Program Care Support and Treatment Services, October 2018. Ministry of Health, Government of India. http://naco.gov.in/sites/default/files/NACO\%20\%20National\%20Technical\%20Guidelines\%20on\%20A RT_October\%202018\%20\%281\%29.pdf [Last cited on 01.11.2019]

[15] Rais N, Jamil HM. Prevalence of needle stick injuries among health care providers. Dow University of Health Science. International Journal of Endorsing Health Science Research 2013;1 (2):73-9. www.aeirc-edu.com [Last cited on 01.11.2019]

[16] Cheng HC, Su CY, Yen AMF, et al. Factors affecting occupational exposure to needlestick and sharps injuries among dentists in Taiwan: a nationwide survey. PLoS One 2012;7 (4):e34911.

[17] Centers for Disease Control and Prevention: NIOSH Publications and Products. Preventing Needlestick Injuries in Health Care Settings (2000-108). http://www.cdc.gov/niosh/docs/2000-108/ [Last cited on 01.11.2019]

[18] Hada V, Saurabh K, Sharma A, et al. Nursing students: a vulnerable health-care worker for needlesticks injuries in teaching hospitals. J Family Med Prim Care 2018;7 (4):717-20.

[19] Mcguire-Wolfe, Christine Michelle, Practices and factors influencing sharps use and safety in a suburban fire department and among emergency medical services personnel. Graduate Theses and Dissertations 2013. http://scholarcommons.usf.edu/etd/4541 [Last cited on 01.11.2019]

[20] Lu IC, Jean MCY, Lin CW, et al. Predictive factors for antiHBs status after 1 booster dose of hepatitis B vaccine. Medicine (Baltimore) 2016;95 (39):e5023.

[21] Sernia S, Ortis M, Antoniozzi T, et al. Levels of anti-HBS antibody in HBV-vaccinated students enrolled in the faculty of medicine, Dentistry and Health Professions of a Large Italian University. BioMed Research International Article ID 712020, 2015;2015:6. https://doi.org/10.1155/2015/712020. [Last cited on 01.11.2019]

[22] Sahana HV, Sarala N, Prasad SR. Decrease in anti-HBs antibodies over time in medical students and healthcare workers after Hepatitis B Vaccination. BioMed Research International Article ID 1327492, 2017;2017:5.

[23] Ouyang B, Li LDX, Mount J, et al. Incidence and characteristics of needlestick injuries among medical trainees at a community teaching hospital: a crosssectional study. J Occup Health 2017;59 (1):63-73.
[24] Sh. Praveen, Devi HS, Ebenezer Phesao E, et al. Needle stick injuries among junior doctors. Indian Medical Gazette 2013: p. 152-6. [Last cited on 01.11.2019]

[25] www.nursingworld.org/MainMenuCategories/ANAMark etplace/ANAPeriodicals/OJIN/TableofContents/Volume 92004/No3Sept04/InjuryPrevention.aspx [Last cited on 01.11.2019]

[26] Foley M, Leyden AT. American Nurses Association independent study module needlestick safety and prevention. [Last cited on 01.11.2019]

[27] Goldstein L, Johnson S. OSHA bloodborne pathogens standard. Implications for the occupational health nurse. AAOHN J 1991;39 (4):182-8.

[28] Centers for Disease Control and Prevention 2008. Workbook for designing, implementing and evaluating a sharps injury prevention program. http://www.cdc.gov/sharpssafety/ [Last cited on 01.11.2019]

[29] The International Health Care Worker Safety Center at the University of Virginia. http://www.healthsystem.virginia.edu/internet/epinet/

[30] Muralidhar S, Singh PK, Jain RK, et al. Needle stick injuries among health care workers in a tertiary care hospital of India. The Indian Journal of Medical Research 2010;131:405-10.

[31] Needlestick and Sharps Injuries. Canadian Centre for Occupational Health and Safety. https://www.ccohs.ca/oshanswers/diseases/needlestic k_injuries.html. \{Last cited on 01.11.2019]

[32] Safer Medical Device Implementation in Health Care Facilities. http://www.cdc.gov/niosh/topics/bbp/safer/ [Last cited on 01.11.2019]

[33] NIOSH Alert: preventing needlestick injuries in health care settings. http://www.cdc.gov/niosh/2000-108.html [Last cited on 01.11.2019]

[34] Bashir H, Qadri SS. A study on needle stick injuries among health care workers in a tertiary care hospital in India. International Journal of Research in Medical Sciences 2019;7 (4):1032-8. Date accessed: 30 Oct, 2019. http://dx.doi.org/10.18203/2320-6012.ijrms20191014

[35] Chakravarthy M, Singh S, Arora A, et al. The epinet data of four Indian hospitals on incidence of exposure of healthcare workers to blood and body fluid: a multicentric prospective analysis. Indian J Med Sci 2010;64 (12):540-8.

[36] Cooke CE, Stephens JM. Clinical, economic and humanistic burden of needlestick injuries in healthcare workers. Medical Devices (Auckl) 2017;10:225-35. 